\title{
Aus der Gutachtenpraxis: Anmerkungen zur Begutachtung einer vermuteten Fensterruptur
}

\section{From the Expert's Office: Remarks for expert evaluation in case of suspected round window membrane rupture}

T. Brusis

\section{Einleitung}

Bei einer hörsturzartig aufgetretenen hochgradigen Innenohrschwerhörigkeit oder Ertaubung wird oftmals eine Ruptur des runden Fensters vermutet, insbesondere wenn der Patient über eine extreme körperliche Anstrengung, insbesondere das Heben einer schweren Last, berichtet hat. Selbst das heftige Nasenschneuzen sowie ein forciertes Valsalva-Manöver wurden schon als Ursache einer Perilymphleckage vermutet [1].

Rupturen des runden Fensters wurden auch in Zusammenhang mit einer Reanimation, einer intensivmedizinisch notwendigen Überdruckbeatmung und CPAP-Maskenbeatmung bei Schlaf-Apnoe beobachtet [3]. Eigene gutachtliche Fälle betreffen z. B. das Anheben einer Rüttelplatte auf eine Ladefläche, das mehrfache Ein- und Ausladen von Reisekoffern durch einen Taxifahrer, das Anschieben einer verklemmten Schiebetür usw.

Als diagnostisch/therapeutische Maßnahme wird dann in den meisten Fällen eine Tympanoskopie mit Inspektion der runden Fensternische und Abdeckung mit einem Bindegewebsstückchen durchgeführt. Die diagnostische Sicherung wird jedoch häufig durch mehrere Umstände erschwert.

Der Einblick in die Rundfensternische ist meist durch eine überhängende Knochenlippe beeinträchtigt. Hier wird verschiedentlich empfohlen, diese mit dem Bohrer oder einem scharfen Löffel abzutragen. Andererseits verbieten sich invasive Maßnahmen bei einer akuten Innenohrpathologie. Der bessere Weg ist dann die endoskopische Untersuchung mit einem kurzen geraden Ohrendoskop, welches auch bei der endoskopischen Tympanoplastik verwendet wird. Wenn aber die Rundfenstermembran nicht eingesehen werden konnte, bzw. wenn der Operateur nicht alle diagnostischen Möglichkeiten ausgenutzt hat, kann eine Ruptur evtl. nur vermutet werden, ist aber nicht nachgewiesen.

Auch der sichere Nachweis einer Fensterruptur mittels eines bildgebenden Verfahrens (MRT, hochauflösendes Felsenbein-CT) ist bisher nicht möglich.

In diesem Zusammenhang muss auch berücksichtigt werden, dass eine Ansammlung freier Flüssigkeit vor dem runden oder ovalen Fenster, welche im Zuge einer Tympanoskopie gesehen wird, keinen Vollbeweis für das Vorliegen einer Fistel darstellt. Nicht selten diffundiert das in den Gehörgang injizierte Anästhetikum in das Mittelohr und kann hier mit Perilymphflüssigkeit verwechselt werden. Eine interessante Innovation zur Fahndung nach Perilymphfisteln stellt der sog. Cochlin-Test dar. Das in Japan entwickelte Testverfahren beruht auf dem ELISA-gestützten intratympanalen Nachweis des Cochlin-Tomo-Proteins (CTP) als perilymphspezifischen Marker [2].

Aus gutachterlicher Sicht ist der überzeugende Nachweis einer Fensterruptur von ausschlaggebender Bedeutung. Das gilt sowohl für die gesetzliche als auch die private Unfallversicherung, in der die Kausalitätsansprüche noch strenger sind. Die versicherungsrechtliche Kausalitätsprüfung hat grundsätzlich in zwei Schritten zu erfolgen.

\section{Schritt}

Zunächst müssen die Tatbestände 1 bis 3 (eine ungewöhnlich belastende Körperanstrengung als Arbeitsunfall (1), eine Fensterruptur (2) und die plötzlich eingetretene Innenohrschwerhörigkeit bzw. Ertaubung (3) im Vollbeweis nachgewiesen werden.

\section{Schritt}

Im zweiten Schritt ist die kausale Verknüpfung zwischen den Tatbeständen 1 bis 3 zu prüfen. Hier reicht dann für einen $\mathrm{Zu}$ sammenhang zwischen den Tatbeständen in der gesetzlichen Unfallversicherung die hinreichende Wahrscheinlichkeit aus, in der privaten Unfallversicherung wäre allerdings die an Sicherheit grenzenden Wahrscheinlichkeit zu fordern ( $\triangleright$ Abb. 1, dieses Schema gilt analog auch für andere Unfall-Beurteilungen).

Bei fehlendem Nachweis einer Fensterruptur gibt es keinen Anlass für eine wohlmeinende Beurteilung zu Gunsten des Versicherten, um ihm in seiner Auseinandersetzung mit seiner Versicherung zu helfen. Es sollte bedacht werden, dass auch der „klassische“ Hörsturz zu einer plötzlichen Ertaubung führen kann, ein Krankheitsbild, welches uns allen aus Praxis und Klinik geläufig ist. Im Übrigen sind gehäufte Fensterrupturen auch bei Gewichthebern nicht bekannt, die unter extremer Anstrengung Langhanteln von enormem Gewicht durch Reißen oder Stoßen zur Hochstrecke bringen. Außerdem führen auch die Presswehen einer Schwangeren nicht in gehäuftem Maße zu einer Fensterruptur. 


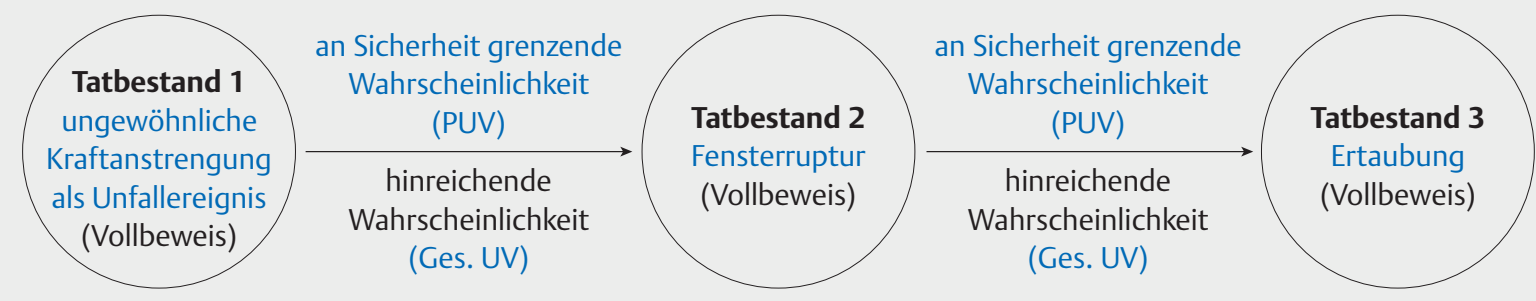

- Abb. 1 Eine Schwerhörigkeit oder Ertaubung kann nur dann mit der für die gesetzliche Unfallversicherung notwendigen hinreichenden Wahrscheinlichkeit auf einen Arbeitsunfall zurückgeführt werden, wenn die vermutete Fensterruptur im Vollbeweis belegt worden ist [2].

\section{Fazit}

Eine plötzlich aufgetretene Innenohrschwerhörigkeit oder Ertaubung kann nur dann auf eine vermutete Fensterruptur zurückgeführt werden, wenn diese im Vollbeweis nachgewiesen ist. Wenn die runde Fensternische aus anatomischen Gründen (vorspringende Knochenlippe) nicht eingesehen werden kann und der Operateur nicht alle diagnostischen Möglichkeiten (Endoskopie) ausnutzt, können diese Umstände nicht als Beweiserleichterung angeführt werden. Es reicht nicht aus, wenn eine Fensterruptur nur für möglich oder für „wahrscheinlich“ gehalten wird. Auch der Hinweis, dass der Operateur die Fensternische prophylaktisch mit einem Bindegewebsstückchen abgedichtet hat, ist kein Beweis dafür, dass tatsächlich eine Fensterruptur vorgelegen hat. Ist aber eine Fensterruptur tatsächlich nachgewiesen, reicht in der ges. Unfallversicherung die hinreichende Wahrscheinlichkeit aus, um einen Zusammenhang mit einer plötzlich eingetretenen Schwerhörigkeit/Ertaubung herzustellen, wenn der Tatbestand 1 (ungewöhnliche Kraftanstrengung) nachgewiesen ist.

\section{Korrespondenzadresse}

Prof. Dr. med. T. Brusis

Institut für Begutachtung

Dürener Str. 199-203

50931 Köln

E-Mail: prof-brusis@t-online.de
Literatur

[1] Comacchio F, Mion M. Sneezing and Perilymphatic Fistula of the Round Window: Case Report and Systematic Review of the Literature. J Int Adv Otol 2018; 14 (1): 106-111

[2] Feldmann H, Brusis T. Das Gutachten des HNO-Arztes. Thieme, 8. Aufl. 2019

[3] Ikezono T, Matsumura $\mathrm{T}$, Matsuda $\mathrm{H}$ et al. The diagnostic performance of a novel ELISA for human CTP(Cochlin-tomoprotein) to detect perilymph leakage. PloS One 2018; 13 (1): e0191498

[4] McCormick JP, Hildrew DM, Lawlor CM et al. Otic Barotrauma Resulting from Continuous Positive Airway Pressure: Case Report and Literature Review. Ochsner J 2016; 16 (2): 146-149

\section{Bibliografie}

DOI https://doi.org/10.1055/a-0954-7260

Laryngo-Rhino-Otol 2019; 98: 636-637

(c) Georg Thieme Verlag KG Stuttgart · New York ISSN 0935-8943 